\title{
GLOBAL SENSITIVITY ANALYSIS OF THE PARAMETERS OF THE MODIFIED UNIVERSAL SOIL LOSS EQUATION
}

\author{
AN, L. S. $.^{*}-$ LIAO, K. H. ${ }^{2}-$ ZHOU, B. H. ${ }^{1}-$ PAN, W. ${ }^{1}-$ CHEN, Q. ${ }^{1}$ \\ ${ }^{I}$ Department of Environmental Science, Anqing Normal University \\ 1318 North Jixian Road, Anqing 246011, P.R. China \\ ${ }^{2}$ Key Laboratory of Watershed Geographic Sciences \\ Nanjing Institute of Geography and Limnology, Chinese Academy of Sciences \\ 73 East Beijing Road, Nanjing 210008, P.R. China \\ (phone: +86-556-5708192; fax: +86-556-5708192) \\ *Corresponding author \\ e-mail: als00316@163.com \\ (phone:+86-556-5708192) \\ (Received $30^{\text {th }}$ Jun 2016; accepted $26^{\text {th }}$ Sep 2016)
}

\begin{abstract}
Global sensitivity analysis of the parameters of the modified universal soil loss equation (MUSLE) was conducted by using the extended Fourier amplitude sensitivity test (EFAST) method. Results show that the runoff factor, slope length and gradient factor, crop management factor and erosion control practice factor were the most sensitive parameters which affected the model outputs, followed by soil texture, organic matter content, aggregation class and the class of the water permeability of the soil profile. The number of iterations for Monte Carlo simulation had significant influence on the sensitivity analysis results. Only when the number of iterations was larger than 50000, EFAST can yield satisfactory convergence of sensitivity indices. Overall, the interaction of the runoff factor, soil erodible factor and adjustment factors was substantial when using the MUSLE. The key parameters should be prioritized for calibration to determine the most optimal values and reduce the uncertainty in soil loss predictions.
\end{abstract}

Keywords: MUSLE equation, EFAST method, key parameters, Monte Carlo simulation, uncertainty analysis

\section{Introduction}

China is one of the most serious soil erosion countries in the world, and the amount of soil erosion is 5 billion tons per year, accounting for nearly $30 \%$ of the total land area. Water loss and soil erosion causes the limited land resources in ruins and soil fertility decline, and frequent flood disasters due to large amounts of sediment discharge. At the same time, it also can influence the social and economic sustainable development of China. To some extent, it is becoming the main ecological environmental problems (Wang et al., 2001). There are many reasons for water loss and soil erosion. The key influencing factors are different in different regions, but in general can be divided into natural factors and human factors (Li et al., 2012). Natural factors include topography, geology, climate, soil, vegetation and other foundation erosion power factor, while human factors are mainly for large-scale destruction of the vegetation and unreasonable land use and other human activities (Bu et al., 2002; Yao et al., 2013). Therefore, in order to prevent water loss and soil erosion effectively, it should practice soil and water conservation on the basis of analyzing these two influencing factors. 
The Prediction of the amount of soil loss is directly related to the layout of the prevention and control measures of soil and water conservation. In order to quantitatively forecast soil loss quantity, the modified universal soil loss equation (MUSLE) was proposed and has been widely used around the world (Meyer, 1984; Toy et al., 1999; Zhang et al., 2009; Arekhi and Rostamizad, 2011; Odongo et al., 2013). MUSLE was improved on the basis of the universal soil loss equation (USLE). In MUSLE equation, rainfall erodible factor was replaced by runoff factor, thus improving the sediment yield prediction (Williams and Berndt, 1977). Soil erodible K value is another important factor in MUSLE, which is decided by a variety of soil physical and chemical properties. It is worth noting that the MUSLE equation is applied under the condition of standard community (22.13 m long slope, 9\% gradient, bare fallow with cultivation up and down the slope). Therefore, MUSLE also introduced three correction factors (slope length and gradient factor, crop cover factor and water conservation measures factor) under different field conditions. It can be seen that soil loss quantity prediction accuracy depends on above-mentioned parameters. However, not all the parameters can be obtained by experimental measurement. Sometimes, we just indirectly estimate them by empirical model or statistical methods. Even the measured value often has observational error due to limited conditions. These factors may lead to strong uncertainty in forecast results.

Sensitivity analysis (SA) is a kind of effective method used to evaluate the uncertain model. Through sensitivity analysis for the parameters of MUSLE equation, the part of sensitive parameters which have greater influence on the predicted results can be screened out. The sensitive parameters can be priority measured, with the limited resources, which has a very good guidance function for popularization and application of the MUSLE equation. SA method includes local sensitivity analysis (or called OAT) and global sensitivity analysis (GSA). The main drawback of the former is that this method can only analyze the effect of individual parameters on the predicted results, and ignore the parameters of the coupling action between indirect effects. The latter GSA method is able to deal with this problem (Saltelli et al., 1999). The typical global sensitivity analysis methods are the Sobol technique (Sobol, 1993) and extended Fourier amplitude sensitivity test (EFAST), which have been applied for land (Wang et al., 2013), plants (Wu et al., 2009; Jiang et al., 2011) and hydrological model (Kong et al., 2011) parameters. This study will use the EFAST method to analyze the sensitivity of the parameters in the MUSLE equation. To our knowledge, little research has been conducted to discuss this issue.

\section{Model and Method}

\section{Modified Universal Soil Loss Equation (MUSLE)}

MUSLE was proposed by Williams and Berndt based on the previous studies in the early 1970 s.

$$
A=\alpha(Q \times q)^{\beta} \times K \times L S \times C \times P
$$

(Where, $A$ is the sediment yield from an individual storm in metric tons; $Q$ is the storm runoff volume in $\mathrm{m}^{3} ; q$ is the peak runoff rate in $\mathrm{m}^{3} \mathrm{sec}^{-1} ; K$ is soil erodible factor in $\mathrm{Mg}$ $\mathrm{MJ}^{-1} \mathrm{~mm}^{-1}$ (Wischmeier et al., 1971); LS is the slope length (dimensionless) and gradient factor (topographical factor) (dimensionless); $C$ is the crop management factor 
(dimensionless); $P$ is the erosion control practice factor (dimensionless); $\alpha$ and $\beta$ are location specific conceptual factors)

$$
K=\frac{2.1 \times 10^{-4} \times(N 1 \times N 2)^{1.14} \times(12-O M)+3.25 \times(R-2)+2.5 \times(U-3)}{100}
$$

(Where, N1 is the sum of fine silt $(0.05 \sim 0.1 \mathrm{~mm})$ and silt $(0.002 \sim 0.05 \mathrm{~mm})$ contents; $N 2$ is the difference between 100 and the clay content $(0 \sim 0.002 \mathrm{~mm}) ; O M$ is organic matter content; $R$ is the coefficient of soil structure level; $U$ is the level in soil permeability coefficient). Substitute Eq.2 into Eq.1:

$$
\begin{aligned}
A= & \alpha(Q \times q)^{\beta} \times\left[2.1 \times 10^{-6} \times\left(N_{1} \times N_{2}\right)^{1.14} \times(12-O M)+3.25 \times 10^{-2} \times(R-2)\right. \\
& \left.+2.5 \times 10^{-2} \times(U-3)\right] \times L S \times C \times P
\end{aligned}
$$

\section{Extended Fourier amplitude sensitivity test (EFAST)}

EFAST is a global sensitivity analysis method proposed by Saltelli in 1999. The characteristics of this method lies in the combination of Sobol and FAST method for their respective advantages, for example, the few number of sample size, high computation efficiency and a good algorithm robustness (Saltelli et al., 1999). The EFAST analyzes the model input parameters generated by the model output variance, and reflects the relative importance (sensitivity) of the parameters as well as the contribution of changes in the model output. The total variance of model was not only derived from the changes of a single impact input parameters, but also considered the interaction between the parameters, which makes it very suitable for solving the "equifinality" phenomenon in numerical simulation. Assuming that the model can be expressed as:

$$
Y=f\left(x_{1}, x_{2}, \ldots, x_{k}\right)
$$

(Where, $Y$ is the model output; $x_{1}, x_{2}, \ldots, x_{\mathrm{k}}$ are independent input factors). Selecting the appropriate periodic function $G_{\mathrm{k}}$, Eq.4 is converted to $Y=f(\mathrm{~s})$, and satisfies:

$$
x_{k}(s)=G_{k}\left(\sin \omega_{k} s\right), \quad \forall k=1,2, \cdots, n
$$

(Where, $s$ is a scalar; $\omega_{\mathrm{k}}$ is the corresponding integer frequency by $x_{\mathrm{k}}$ ). EFAST uses Fourier transform to decompose the $f(\mathrm{~s})$ :

$$
f(s)=\sum_{p=-\infty}^{+\infty}\left|A_{p} \cos p s+B_{p} \cos p s\right|
$$

(Where, $p$ is the Fourier transform parameter; $A_{\mathrm{p}}$ and $B_{\mathrm{p}}$ are the Fourier amplitudes). The sampling values use $G_{\mathrm{k}}$ conversion for each parameter value and the input model for multiple operations can calculate model of total variance by means of taking sample in 
the interval of $[-\pi, \pi]$. The concrete method and process have been introduced in the document. Because there has interaction between single parameters, we can resolve $V$ as:

$$
\begin{gathered}
V=\sum_{i} V_{i}+\sum_{i \neq j} V_{i j}+\cdots+V_{12 \cdots k} \\
\left\{\begin{array}{c}
V_{i}=V\left[E\left(Y \mid x_{i}\right)\right] \\
V_{i j}=V\left[E\left(Y \mid x_{i}, x_{j}\right)\right]-V_{i}-V_{j}
\end{array}\right.
\end{gathered}
$$

(Where, $V_{\mathrm{i}}$ and $V_{\mathrm{j}}$ are variance caused by parameter $x_{\mathrm{i}}$ and $x_{\mathrm{j}}$ respectively; $V_{\mathrm{ij}}$ is the coupled variance from $x_{\mathrm{j}}$ impacting on $x_{\mathrm{i}} ; V_{1,2 \ldots, \mathrm{k}}$ is variance produced by $x_{1,2, \ldots, k}$ acting on $x_{i}$; $E$ is conditional expectation). By normalization processing, parameter $x_{i}$ 's first order sensitivity index $S_{\mathrm{i}}$ can be defined as:

$$
S_{i}=V_{i} / V
$$

Similarly, parameter $x_{\mathrm{i}}$ 's second order sensitivity index $S_{\mathrm{ij}}$ and total sensitivity $S_{\mathrm{Ti}}$ index can express respectively:

$$
\left\{\begin{array}{c}
S_{i j}=V_{i j} / V \\
S_{T i}=S_{i}+S_{i j}+\cdots+S_{12 \cdots k}
\end{array}\right.
$$

(Where, $S_{\mathrm{i}}$ is the direct contribution rate of parameter $x_{\mathrm{i}}$ on $V$, while $S_{\mathrm{Ti}}$ reflects the amount of between the direct contribution rate and the coupling effect between parameters). $S_{\mathrm{Ti}}$ equals to $S_{\mathrm{i}}$ if there are no coupling of variance. In this case, the EFAST analysis is the same as the local sensitivity. This research compares each parameter index that can analyze the impact of which on input MUSLE model.

\section{Experimental Design}

The research object of this study is to analyze the sensitivity of all 12 parameters in MUSLE model, and use EFAST method to quantitatively identify key influencing parameters. Runoff data adopted in this study are from the Kenya Government Water Resource Management Authority (WRMA)'s observational data, which were collected on Malewa Basin in 2007 (Odongo et al., 2013). Ranges of variation of the four runoff factors $(\alpha, \beta, Q$ and $q)$ are shown in Table 1 . The range of five factors affecting $\mathrm{K}$ value $(N 1, N 2, O M, R$ and $U$ ) is in strict accordance with the applicable conditions of monogram formulas. In addition, the ranges of three correction factors $(L S, C$ and $P)$ are reasonably limited by prior knowledge (Table 1 ). When determining a reasonable parameter range, we firstly assumed that they are evenly distributed between the minimum and maximum values. Then, the Monte Carlo method was used to randomly sample all parameters. In order to make sampling fill the entire parameter space, we set the sampling frequency to 500000 times. Subsequently, the parameters were successively entered into the MUSLE equation and calculate, and then we can get the stochastic simulated values of the amount of soil loss and statistical variance. Finally, the 
EFAST method was applied to calculate first-order sensitivity index and total sensitivity index of every parameter, which were then compared.

Table 1. The data range of parameters in MUSLE equation

\begin{tabular}{ccc}
\hline Parameters & Minimum value & Maximum value \\
\hline$\alpha$ & 1 & 65 \\
$\beta$ & 0 & 1 \\
$Q / \mathrm{m}^{3}$ & 0 & 30 \\
$q /\left(\mathrm{m}^{3} / \mathrm{s}\right)$ & 0 & 3 \\
$N 1 / \%$ & 0 & 70 \\
$N 2 / \%$ & 0 & 100 \\
$O M / \%$ & 0 & 4 \\
$R$ & 1 & 4 \\
$U$ & 1 & 6 \\
$L S$ & 0 & 20 \\
$C$ & 0 & 1 \\
$P$ & 0 & 1 \\
\hline
\end{tabular}

\section{Results and Discussion}

\section{The first-order sensitivity index}

As can be seen from Figure 1, when not considering the interactions among the parameters, the first-order sensitivity index of $\beta$ is about 0.06 , which is significantly larger than those of the other parameters of the model. This suggests that the MUSLE model is most sensitive to the changes in $\beta$, which contributes $6 \%$ of the model output variance. Followed by the parameters $P$ and $\alpha$, their first-order sensitivity indexes are close to 0.04 . The first-order sensitivity indices of $N_{1}, N_{2}$ and $L S$ (between 0.02 and 0.03 ) are smaller than those of other parameters, while the remaining parameters' first-order sensitivity index vary between 0 and 0.02 , indicating that their effects on model output is relatively small. It is worth noting that the sum of all first-order sensitivity index parameters is only 0.29, indicating that their contributions to the total variance of the model output are $29 \%$. This implies that there is still $71 \%$ of the variance is not taken into account. This may be contributed to two reasons: the first is MUSLE model's nonlinear relationship between input and output, and the second is the coupling between parameters.

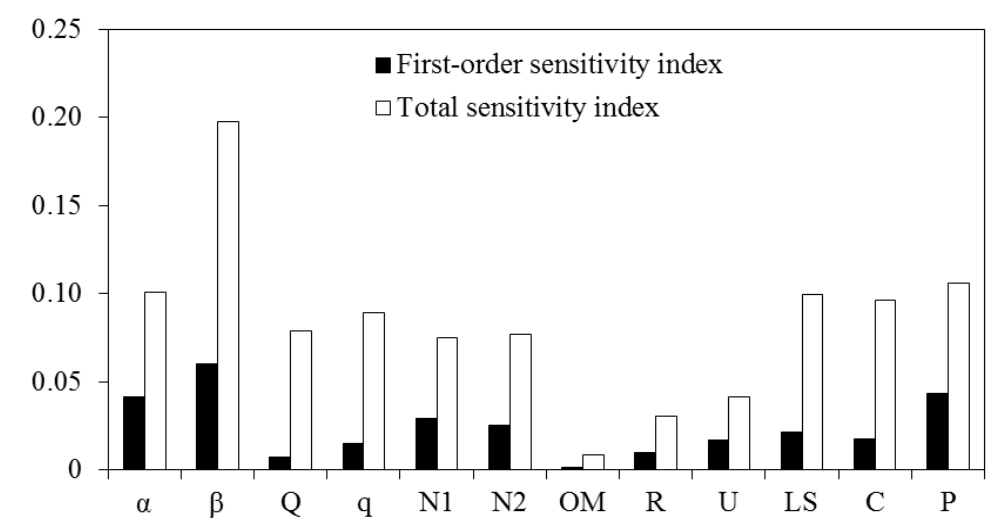

Figure 1. Parameter sensitivity index in MUSLE equation 
In previous studies, Odongo and other scholars have used OAT method to conduct a partial sensitive analysis on four runoff factors $(\alpha, \beta, Q$ and $q)$ in MUSLE equation. They found that compared with the other two parameters, the amount of soil loss predicted value is more sensitive to the changes in location factors $\alpha$ and $\beta$ (Odongo et al., 2013), which is consistent with the findings of this study. This also demonstrated the feasibility of applying EFAST method in MUSLE model. In addition, based on a lot of soil data analysis of Bulgaria area, Mitova and Rousseva (2013) used sensitivity analysis method to study the impact factors of soil erodible factor $K$. They found that $K$ values is most sensitive to N1 and N2 changes, which is consistent with the conclusions of this study.

\section{The total sensitivity index}

The total sensitivity index can be used to represent the coupling effect among parameters (Figure 1). The total sensitivity index of parameter $\beta$ is 0.20 , which increases significantly when compared with the first-order sensitivity index. It is significantly greater than those of other parameters. Followed by parameters $P, \alpha, L S, C$ and $q$ successively, varying from 0.09 to 0.11 . The parameters $Q, N 1$ and $N 2$ are relatively close to each other and their total sensitivity index is approximately 0.08 , while the other parameters' contribution to model output is relatively small. It can be deduced that the order of every parameter sensitivity have changed when considering the coupling between parameters. The reason may be that the interaction between different parameters is an objective reality. In this case, the total sensitivity index can better reflect the model output's relative importance for each parameter.

The above results indicate that the parameter $\beta$ is the key influencing parameter, which may be related to that this parameter exists in the form of exponential power in the MUSLE equation. Meanwhile, it shows that we should give priority to correct the parameters to obtain the optimum value when calculating the amount of soil loss in specific areas. In particular, in the powerful hydrological model SWAT, the default $\beta$ value is 0.56 , while the location factor $\alpha$ is 11.8. These values are obtained from foreign soil and hydrological surveys. This will cause great prediction error or uncertainty if they are directly used in China. Therefore, it must be corrected in practical application. $L S, C$ and $P$ also have a great influence on the model output, which is consistent with our previous understanding. $L S$ represents the terrain information, which can directly affect the washing ability of slope runoff, while $C$ and $\mathrm{P}$ reflect the interactions among the vegetation, crops planting, tillage measures, rainfall distribution and so on. These parameters have a direct or indirect relationship with soil erosion. Compared with the first-order sensitivity index, $Q$ and $q$ 's total sensitivity indices increase significantly. This is because they reflect the combined effects of precipitation, rainfall duration, rainfall intensity and surface runoff on soil erosion. $N 1$ and $N 2$ 's total sensitivity indices also increase obviously, because they represent the soil texture information and dynamic factors. Soil erosion is closely related to soil water holding capacity and water transmission capacity. It is worth noting that the impact of soil organic matter on the model output is not always obvious. The reason may be that the $O M$ content is relatively small in this study.

\section{The number of Monte Carlo sampling's influence}

In order to investigate the effect of the number of samples on EFAST, this study respectively conduct different numbers $(1000,5000,10000,50000$ and 100000$)$ of 
random sampling. For comparative analysis, assuming that the results from the sampling number of 500000 is approximately correct. As can be seen in Figure 2, when the number of samples is less than 50000 times, every parameter's first-order sensitivity index and total sensitivity index of vary significantly with the sampling numbers. When the sampling number is larger than 50000 , the results did not vary. This suggests that the number of Monte Carlo sampling has a great impact on the EFAST analysis results. If the sampling number is very small, the model's output is not representative and accurate. Meanwhile, the excessive samples will result in the time increases exponentially and even increase the simulating costs. Therefore, when using EFAST model, sampling number should be carefully selected. It is better to determine the value by trial and error analysis.
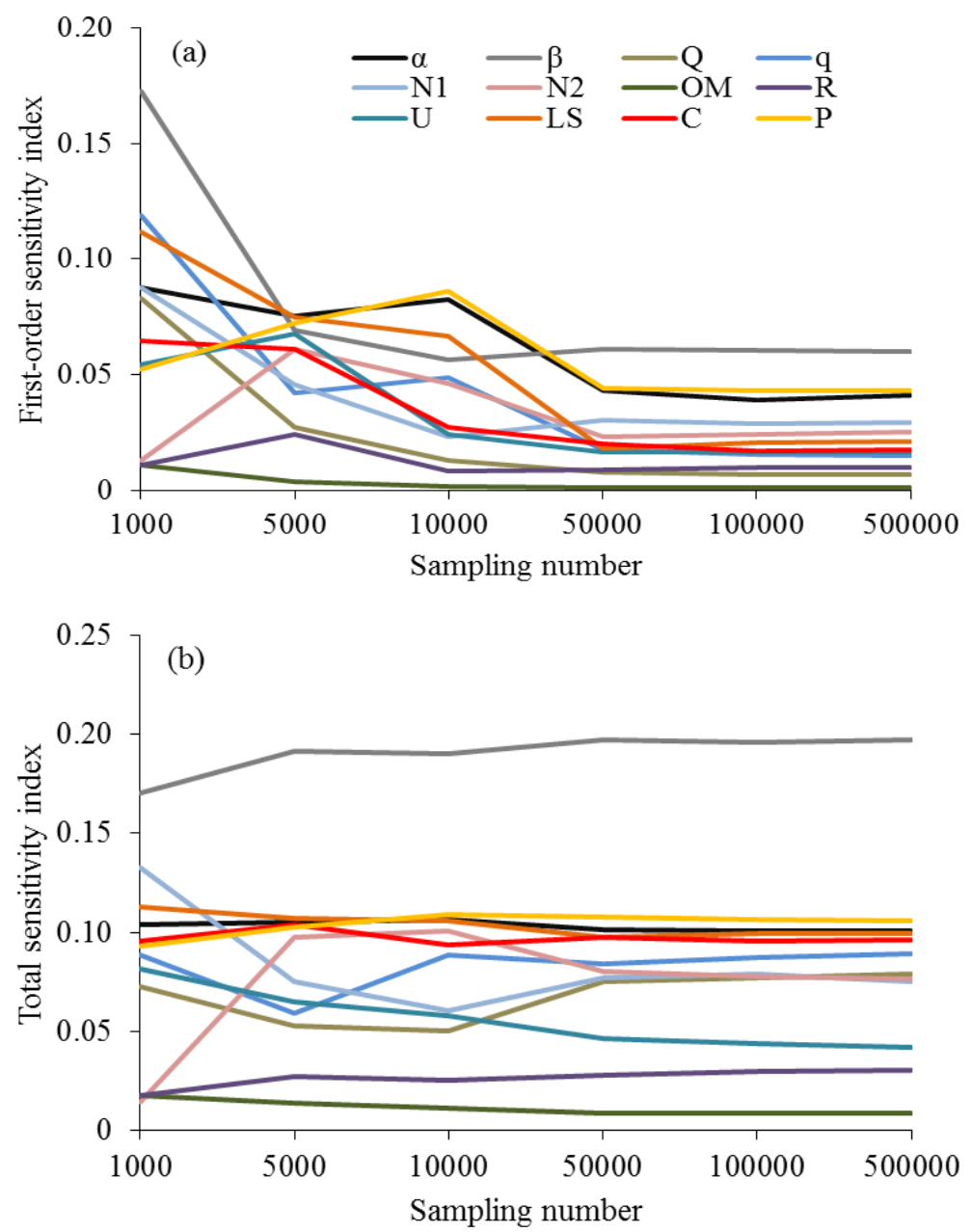

Figure 2. Effects of different Monte Carlo sampling frequency to EFAST analysis

\section{The impact of various parameters' changes}

Runoff and correction factors represent the external causes of soil erosion, while the $K$ value is internal reason. They do not always change at the same time in the regionalization applications of MUSLE equation. Therefore, it is necessary to carry out EFAST analysis on these factors respectively, which helps to further clarify the influence of various parameters on MUSLE model output. In order to independently analyze the 
sensitivity of runoff and correction factors, $K$ value should be set to a constant, which is defined as 0.2 in this study. When the independent analysis of the influence factors of $K$ value, the remaining parameters are defined as 1 .

From the Figure 3, when the $K$ value is defined, the most sensitive parameter of MUSLE model is $\beta$. The Monte Carlo sampling number's influence is 0.24 . This is the same as all parameter in EFAST's analysis. However, the next parameter sensitivity priority is different, followed by $P, L S, C, \alpha, q$ and $Q$, and varies from 0.11 to 0.14 . Under the condition of runoff and correction factors being determined, Sensitivity sequencing for 5 impact parameters of $K$ value consistent with previous analyzes, but their impact on the model output varies. Among them, N1 and N2 are the most sensitive. These two parameters' total sensitivity indices are close to 0.40 , showing that they can explain $80 \%$ the prediction of the results of variance. Also, it shows that other three parameters have little influences on the output of the model.

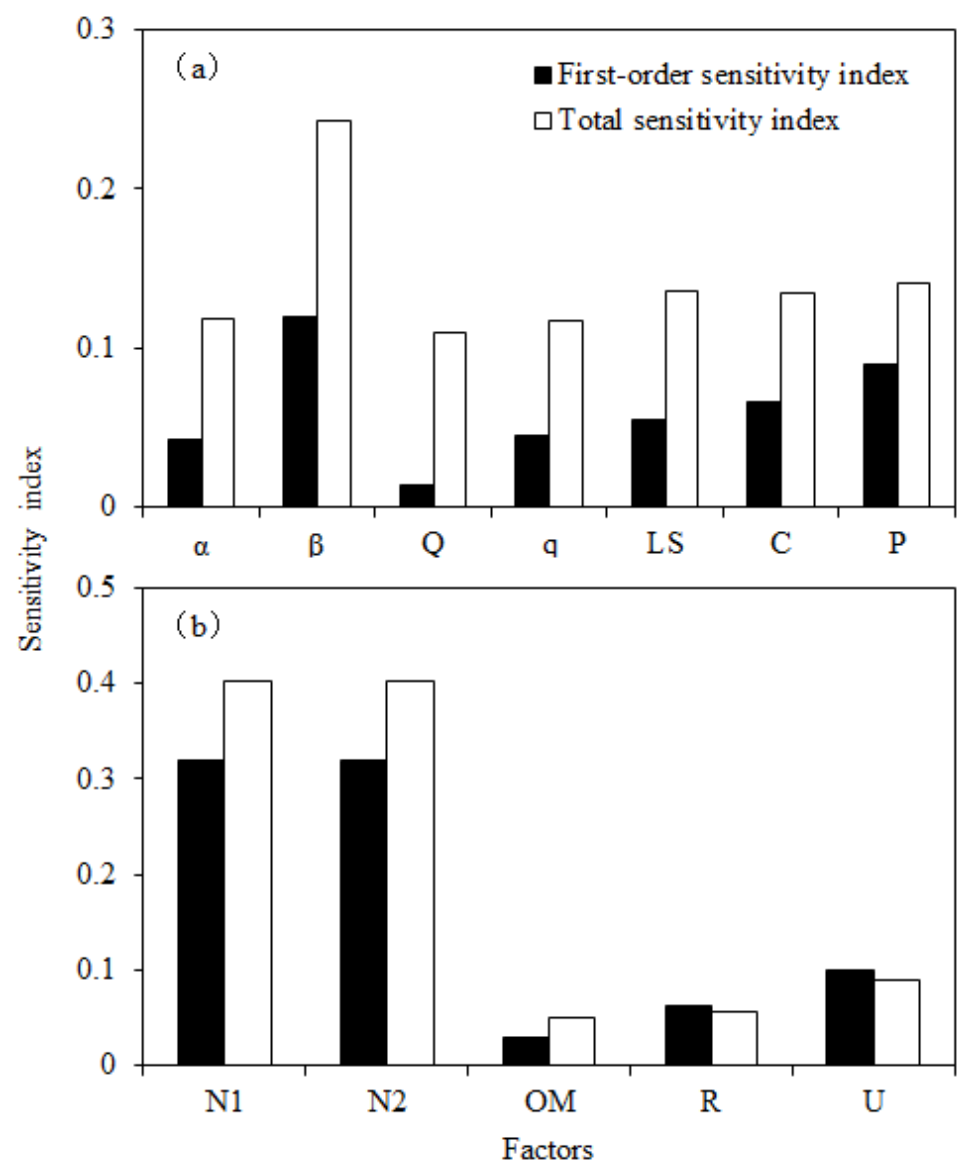

Figure 3. Sensitivity index of runoff and correction factors (a) and influencing factors of $K$ value (b)

The analysis results (Figures 1 and 3) show that the interaction between runoff factor, $K$ value and correction factor should be considered in the application of MUSLE equation. The key point is to optimize the key parameters of the model output, so as to reduce the uncertainty of the prediction results and improve the application ability of the MUSLE model. Generally speaking, soil texture is a very stable property and easy to get with high precise. However, the progress of runoff has much more uncertainty and the 
measurement of this variable is rather difficult. Especially, location factors $\alpha$ and $\beta$ can only be obtained by adjusting the model. Therefore, under the limited resources, the prediction result uncertainty caused by the difference of runoff factor should be considered more. In addition, the effect of correction factor is also great. Especially the secondary factors of $P$ factor are varied, and it is difficult to quantify. The fine expression of $C$ value also has certain difficulty, usually based on experience. So we should be caution about their value in order to ensure that it is scientific. The calculation of $L S$ depends on the accuracy of DEM. Meanwhile, different terrain exponentiation algorithm will influence this value. So we should consider different DEM accuracy or algorithms to select best parameters for ensuring relative rationality and exactitude in the future.

\section{Conclusions}

(1) The location factor $\beta$ is the most sensitive parameter to predict the amount of soil loss, followed by $P, \alpha, L S, C$ and $q, K$ 's influencing factors (such as $O M, R$ and $U$ ) is less sensitive, So the accuracy of runoff and correction factors should be determined firstly in simulated calculation.

(2) The Monte Carlo sampling frequency has a significant impact on EFAST's analytical results. The difference of each parameter's sensitive index is significant when sampling number is less than 50000 times. When the sampling number is more than 50000 , the results are close to each other. However, this value (50000) is not representative of other similar studies. Therefore, a reasonable sampling number should be selected by trial and error analysis in order to ensure the sampling efficiency.

(3) Each parameter's first-order sensitivity index is different from the total sensitivity index. It shows that the traditional partial sensitivity analysis methods ignore the coupling effects of parameters, while the global sensitivity analysis can overcome these problems effectively.

Acknowledgements. This study was financially supported by the project of Natural Science Research for Colleges and Universities of Anhui Province, China (grant KJ2016A424, AQKJ2014B022)

\section{REFERENCES}

[1] Wang, X. K., Ouyang, Z. Y., Xiao H., Fu B. J. (2001): Distribution and division of sensitivity to water-caused soil loss in China. - Acta Ecologica Sinica 21(1): 14-19.

[2] Li, W. J., Wang X. K., Li D. X., Yang, S. F. (2012): A physically-based distributed watershed water erosion prediction model. - Shui Li Xue Bao 43(3): 264-274.

[3] Bu, Z. H., Yang L. Z., Bu Y. H. Wu, J. Y. (2002): Soil erodibility (K) value and its application in Taihu lake catchment. - Acta Pedological Sinica 39(3): 296-300.

[4] Yao, W. Y., Ran, D. C., Chen, J. N. (2013): Recent change in runoff and sediment regimes and future projections in the Yellow River basin. - Advances in Water Science 24(5): 607-616.

[5] Meyer, L. D. (1984): Evolution of the universal soil loss equation. - Journal of Soil and Water Conservation 39: 99-104.

[6] Toy, T. J., Foster, G. R., Renard, K. G. (1999): RUSLE for mining, construction and reclamation lands. - Journal of Soil and Water Conservation 54(2): 462-467. 
[7] Zhang, Y., Degroote, J., Wolter, C., Sugumaran, R. (2009): Integration of modified universal soil loss equation (MUSLE) into a gis framework to assess soil erosion risk. - Land Degradation \& Development 20(1): 84-91.

[8] Arekhi, S., Rostamizad, G. (2011): Sediment yield estimating from three micro-watersheds by integrated KW-GIUH and MUSLE models. - Advances in Environmental Biology 5(6): 1346-1358.

[9] Odongo, V. O., Onyando, J. O., Mutua, B. M., Oel, P. R. van., Becht, R. (2013): Sensitivity analysis and calibration of the Modified Universal Soil Loss Equation (MUSLE) for the upper Malewa Catchment, Kenya. - International Journal of Sediment Research 28: 368-383.

[10] Williams, J. R., Berndt, H. D. (1977): Sediment yield prediction based on watershed hydrology. - Transactions of the ASAE 20(6): 1100-1104.

[11] Saltelli, A., Tarantola, S., Chan, K. P. S. (1999): A quantitative model-independent method for global sensitivity analysis of model output. - Technometrics 41(1): 39-56.

[12] Sobol, I. M. (1993): Sensitivity analysis for non-linear mathematical methods. - Mathematical Modeling and Computational Experiment 1: 407-414.

[13] Wang, J. D., Guo, W. D., Li, H. Q. (2013): Application of extended Fourier amplitude sensitivity test (EFAST) method in land surface parameter sensitivity analysis. - Acta Physica Sinica 62(5): 438-445.

[14] Wu, J., Yu, F. S., Chen, Z. X., Chen, J. (2009): Global sensitivity analysis of growth simulation parameters of winter wheat based on EPIC model. - Transactions of the CSAE 25(7): 136-142.

[15] Jiang, Z. W., Chen, Z. X., Zhou, Q. B. (2011): Global sensitivity analysis of CERES-Wheat model parameters. - Transactions of the CSAE 27(1): 236-242.

[16] Kong, F. Z., Song, X. M., Zhan, C. S., Ye A. Z. (2011): An efficientquantitative sensitivity analysis approach for hydrological model parameters using RSMSobol method. - Acta Geographica Sinica 66(9): 1270-1280.

[17] Wishmeier, W. H., Johnson, C. B., Cross, B. V. (1971): A soil erodibility nomograph for farmland and construction sites. - Journal of Soil and Water Conservation 26(5): 189-193.

[18] Mitova, M., Rousseva, S. (2014): Sensitivity analysis of the USLE soil erodibility factor to its determining parameters. - Geophysical Research Abstracts 16: EGU2014-650-2. 